\title{
Challenges in cancer molecular targets and therapeutics
}

\section{Yisong Wang and Giuseppe Giaccone*}

National Cancer Institute, National Institute of Health, Bethesda, MD, USA

*Correspondence: giacconeg@mail.nih.gov

Efforts of cancer research have yielded significant advance in our understanding on the complexity of cancer during the past several decades. It is generally accepted that cancer development is a multi-step and multigenic event (Hahn and Weinberg, 2002). The hallmarks of cancer comprise a series of genetic and epigenetic gainand loss-of-functions of oncogenes and tumor suppressor genes, respectively, that render cancer cells capable of unrestricted replication potential, resistance to proapoptotic stimuli, sustained angiogenesis, self-sufficiency in growth signals, insensitivity of growth suppressor signals, evasion of immune surveillance, energy metabolism reprogramming, and acquisition of tumorpromoting inflammation (Hanahan and Weinberg, 2011). With the explosion of knowledge on genes and molecular pathways that govern those cancer hallmarks, mechanism-based target-specific therapies have been developed. The rapidly emerging role of targeted therapies may be considered one of the most remarkable developments in the field of cancer research and therapeutics over the past several decades.

Most of the target-specific drugs have hitherto been designed to cripple proteins or molecular pathways that are thought to be the Achilles' heel of cancer. If the targeted genes or pathways are genuinely indispensable for the tumors, their inhibition should impede tumor propagation and progression. The target-specific "smart bombs" in theory should discriminatingly destroy cancer cells and leave the normal cells untouched. The very first two drugs of this kind are trastuzumab/Herceptin, a humanized monoclonal antibody against HER2/neu receptor (Baselga et al., 1998) and Imatinib, a BCR-ABL inhibitor (Druker et al., 2001). CML patients with BCR-ABL fusion protein and metastatic breast cancer patients with HER2/neu amplification showed improved response and survival rates to imatinib and trastuzumab respectively (Baselga et al., 1998; Druker et al., 2001). Unfortunately, following initial promising responses, resistance is often inevitable due to mechanisms, such as secondary mutations of the targets (Gorre et al., 2001) and activation of bypass pathways (Jones and Buzdar, 2009). A thorough understanding of the resistance mechanisms will facilitate the development of more effective drugs.

In the recent past, targeting addictive oncoproteins, such as receptor tyrosine kinases, has overwhelmingly led drug development in the field of cancer targetspecific therapies. The concept of targeting non-oncogene addiction has been investigated as an alternative anti-cancer therapy (Luo et al., 2009). Proteins, such as protein chaperone Hsp90 and proteasomes, themselves are not oncogenes as they have rarely been found mutated or amplified in tumor cells. Many oncogenic proteins are often over-produced, unfolded or misfolded due to their amplification, mutations, abnormal epigenetic, or post-translational modifications, etc., in tumor cells. As a result, cancer cells become addictive to Hsp90 and proteasomal functions for survival as the latter are required to fold the misfolded oncoproteins, which otherwise will be destroyed through the ubiquitination-dependent proteasome degradation pathways (Whitesell and Lindquist, 2005). Many Hsp90 and proteasome inhibitors have been generated and showed some inhibitory effects on various cancer types in preclinical and clinical settings (Rajkumar et al., 2005; Trepel et al., 2010). More potent and less toxic inhibitors are being actively pursued. A major challenge in this field will be to identify the right cancer patients whose cancer cell survival is Hsp90- or proteasome-dependent, and to hit the targets right and hard in time with those inhibitors.

The concept of "metabolic reprogramming or transformation" has emerged as the 7th hallmark of cancer during the past decade (Hanahan and Weinberg, 2011). Many studies suggest that the metabolic reprogramming is required for cancer cell survival and thus might be a good target for anti-cancer therapies (Tennant et al., 2010). The observation that cancer cells show increased rate of glycolysis can be traced back to as early as 1920s (Warburg, 1923). Increased glycolysis under both aerobic and anaerobic conditions promotes diversion from intracellular glucose to pyruvate, ATP, and NADH that in turn facilitate biosynthesis of nucleosides and amino acids required for rapid cancer cell growth and survival. Since most of the enzymes and proteins in the glycolysis pathway are ubiquitously expressed in the body, caution must be paid to selectively target tumor-specific proteins or enzyme isoforms in metabolic addictionbased target-specific therapy. Glucose transporter 1 (GLUT1) which is upregulated and promotes glucose import into the cells, and hexokinase which controls the first step of glycolysis and is upregulated by both HIF and Myc in many cancer types, have been explored as targets for anti-cancer therapies (Tennant et al., 2010). Challenge remains as to the identification of tumor type specific metabolic pathways so that bona fide tumor-specific targets may be used to develop more efficacious and less toxic drugs for metabolic addiction-based targetspecific therapies.

Different molecular networks can steer or buffer overlapping cellular processes, and disruption of one network can lead to an acquired dependency on another. An example of this is synthetic lethal interaction described in yeast and fruit fly, in which perturbation of two genes causes cell death (synthetic lethal) whereas perturbation of either gene alone exerts minimal or no effect on cell survival (Lucchesi, 1968; Hartman et al., 2001). With the discovery of RNA interference (RNAi), the synthetic lethal interaction idea has recently been adopted for unbiased screen for synthetic lethal partners of frequently mutated but pharmacologically non-inhibitable genes, such as Ras, or tumor suppressor genes (PTEN, P53, VHL, APC, BRCA, etc.) that are deleted in tumors and thus cannot be targeted (Kaelin, 2005; Kuiken and Beijersbergen, 2010). This approach will undoubtedly lead to the discovery of novel drug targets and mechanisms of network addiction in cancer cells. It may also open an alternative way 
to effectively treat tumors carrying those "non-druggable" genes. Another attractive aspect of synthetic lethality screen is that it may accelerate the search for regulators of target-specific inhibitor resistance in cancer cells. Erlotinib-treated cancer cells have been subjected to synthetic lethal screen with a library containing $>600$ selected siRNAs against putative EGFR interacting proteins. Inhibition of PRKC, Aurora A, and Stat3 were found synthetic lethal with erlotinib in many erlotinib-resistant cell lines, indicating they may be potential regulators of resistance and have significant clinical implications in EGFR-targeted therapy (Astsaturov et al., 2010). The advancement of synthetic lethal screen has fueled the expectation toward new discoveries of novel biomarkers, targets, and molecular pathways for cancer diagnosis and therapy. To meet these expectations, in the years to come, we wish to see the improvement of not only RNAi technologies but also highthroughput target validations to eliminate false positive discoveries.

Increasing evidence suggests that cancer stem cells (CSCs) are more resistant than other cells to conventional radiotherapy, chemotherapy and most of the targetspecific therapies, though many aspects of CSC hypothesis remain speculative and are still evolving (Zhou et al., 2009). Potential mechanisms of the resistance can be attributed to the observation that CSCs are in quiescent state (Konopleva and Jordan, 2011), resistant to DNA damage and express high levels of $\mathrm{ABC}$ drug pumps and antiapoptotic proteins (Zhou et al., 2009). CSCs that have not been eradicated can lead to recurrence of cancer later. Thus, regimens or drugs that eliminate CSCs independent of cell cycle status may be an attractive strategy in drug development, such as targeting gene products and pathways, for example, the Hedgehog, Notch, and Wnt pathways among others that are activated in CSCs (Konopleva and Jordan, 2011). Based on data derived from a mouse model of human acute myeloid leukemia stem cells, it has been proposed that induction of leukemia stem cell cycle entry before treatment with conventional chemotherapy may be feasible (Saito et al., 2010). Elucidation of the molecular mechanisms underlying the maintenance of CSC quiescence will undoubtedly facilitate the future CSC target therapies. Increasing bodies of evidence suggest that CSC niche, composed of stromal cells, blood vessels, and extracellular matrix components, plays crucial roles in controlling CSC self-renewal, differentiation, epithelial-mesenchymal-transition, invasion, and metastasis (Borovski et al., 2011). Future CSC target therapy should include targeting and blocking of CSC niche signaling and interaction with CSCs for more effective eradication of CSCs (Borovski et al., 2011).

Other areas under fierce development in the field of target-specific therapeutics include exploration of innovative strategies to reactivate tumor suppressors and inhibit oncogenic transcription factor complexes. An example of the former is the discovery and characterization of small molecules that reverse the epigenetic silencing of RASSF1A tumor suppressor protein in preclinical models (Sheikh et al., 2010). Unlike enzymes, transcriptional factors are generally entailed "undruggable". Strategies to disrupt protein-protein interactions and post-translational modifications of oncogenic transcription factor complexes have drawn significant attention recently (Gorczynski et al., 2007; Erkizan et al., 2009). Last but not least, there are great demands for innovative ideas to develop novel vehicles carrying drugs specifically into the tumors and more efficiently through blood brain barriers.

It is disappointing to concede that resistance is almost a universal rule following initial encouraging response in most of the targeted therapeutic trials to date. One of the potential mechanisms of resistance is the presence of dormant tumor cells since most of the radio-, chemo-, and targettherapies rely on cell division and work only against replicating but not quiescent cells (Konopleva and Jordan, 2011). Another possibility is that multiple partially redundant pathways may prevail in tumor cells, and when one pathway is inhibited, tumor cells adopt other redundant pathway(s) to sustain their growth (Hanahan and Weinberg, 2011). The third possibility is that cancer cells may be constantly evolving and target-specific inhibition of one pathway leads to de novo activation of another pathway(s) that keep(s) cancer cells alive. Still other mechanisms of resistance may be attributed to differences in pharmacogenomics, metabolism, and intratumoral drug exposure among individual patients.
With this in mind, understanding cancer molecular networks, heterogeneity, and the nature of cancer evolution will be of paramount importance in directing future target-specific therapeutics and evading resistance.

One of the most formidable challenges in anti-cancer drug development in the past decade has been the amazing number of clinical drug candidates that have emerged. Having phase I testing of all these drugs has become a major hurdle and prioritization has therefore become essential. A typical example is the field of tyrosine kinase inhibitors with inhibitors that target several similar and partially overlapping pathways. Furthermore, the presence of multiple genetic alterations in common tumors, that can act as oncogenic drivers, clearly points to the necessity of using combinations of multiple drugs with multiple mechanisms of action, with drugs that should potentially have little overlapping toxicities. The use of more predictive preclinical models to test single agents and combinations has been partially addressed by genetically engineered mouse models and orthotopic models, but we are still far from optimal prediction of efficacy in humans. Changing in design of phase I trials, with extension phases to assess preliminary activity in enriched populations, the increased use of randomized phase II studies before embarking in phase III testing and the introduction of biomarkers early in development, in order to select patient populations more prone to respond to treatment, all have been taken place at a very rapid pace in recent years. With the rapid growing momentum of new technologies, such as the next-generation sequencing, proteomics, and functional genomics, that allow to examine every molecule of a cancer cell at unprecedented speed and cost-effective manner, it is foreseeable that knowledge on molecular networks and heterogenic regulations of cancer will be the next wave of explosion. Cancer target-specific therapies will heavily rely on the innovative ideas and technologies to uncover the real Archille's heel of cancer. In the next several decades to come, we envision that combination of mechanism-based discretionary targeting of several core pathways vital for tumor survival may bear significant fruits in curing cancers and evading drug resistance. We anticipate that we will be able to find much 
better cancer specific biomarkers for early diagnosis and prognostic prediction, and to treat each patient based on his/her own tumor-specific molecular identities.

This journal is open to contributions in the field discovery of cancer target-specific treatments, some of which have been briefly described above. The journal will be an open forum for the publication of novel strategies for cancer treatment and novel technologies to identify and characterize potential new targets.

\section{ACKNOWLEDGMENT}

The work was supported by the intramural research program of National Cancer Institute, National Institute of Health.

\section{REFERENCES}

Astsaturov, I., Ratushny, V., Sukhanova, A., Einarson, M. B., Bagnyukova, T., Zhou, Y., Devarajan, K., Silverman, J.S., Tikhmyanova, N., Skobeleva, N., Pecherskaya, A., Nasto, R. E., Sharma, C., Jablonski, S. A., Serebriiskii, I. G., Weiner, L. M., and Golemis, E. A. (2010). Synthetic lethal screen of an EGFR-centered network to improve targeted therapies. Sci. Signal. 3, ra67.

Baselga, J., Norton, L., Albanell, J., Kim, Y. M., and Mendelsohn, J. (1998). Recombinant humanized antiHER2 antibody (Herceptin) enhances the antitumor activity of paclitaxel and doxorubicin against HER2/ neu overexpressing human breast cancer xenografts. Cancer Res. 58, 2825-2831.

Borovski, T., De Sousa, E. M. F., Vermeulen, L., and Medema, J. P. (2011). Cancer stem cell niche: the place to be. Cancer Res. 71, 634-639.

Druker, B. J., Talpaz, M., Resta, D. J., Peng, B., Buchdunger, E., Ford, J. M., Lydon, N. B., Kantarjian, H., Capdeville, R., Ohno-Jones, S., and Sawyers, C. L. (2001). Efficacy and safety of a specific inhibitor of the BCR-ABL tyrosine kinase in chronic myeloid leukemia. N. Engl. J. Med. 344, 1031-1037.
Erkizan, H. V., Kong, Y., Merchant, M., Schlottmann, S., Barber-Rotenberg, J. S., Yuan, L., Abaan, O. D., Chou, T. H., Dakshanamurthy, S., Brown, M. L., Uren, A. and Toretsky, J. A. (2009). A small molecule blocking oncogenic protein EWS-FLI1 interaction with RNA helicase A inhibits growth of Ewing's sarcoma. Nat. Med. 15, 750-756.

Gorczynski, M. J., Grembecka, J., Zhou, Y., Kong, Y., Roudaia, L., Douvas, M. G., Newman, M., Bielnicka, I., Baber, G., Corpora, T., Shi, J., Sridharan, M., Lilien, R., Donald, B. R., Speck, N. A., Brown, M. L., and Bushweller, J. H. (2007). Allosteric inhibition of the protein-protein interaction between the leukemiaassociated proteins Runxl and CBFbeta. Chem. Biol. $14,1186-1197$.

Gorre, M. E., Mohammed, M., Ellwood, K., Hsu, N., Paquette, R., Rao, P. N., and Sawyers, C. L. (2001). Clinical resistance to STI-571 cancer therapy caused by BCR-ABL gene mutation or amplification. Science $293,876-880$

Hahn, W. C., and Weinberg, R. A. (2002). Modelling the molecular circuitry of cancer. Nat. Rev. Cancer 2, 331-341.

Hanahan, D., and Weinberg, R. A. (2011). Hallmarks of cancer: the next generation. Cell 144, 646-674.

Hartman, J. L. IV, Garvik, B., and Hartwell, L. (2001). Principles for the buffering of genetic variation. Science 291, 1001-1004.

Jones, K. L., and Buzdar, A. U. (2009). Evolving novel anti-HER2 strategies. Lancet Oncol. 10, 1179-1187.

Kaelin, W. G. Jr. (2005). The concept of synthetic lethality in the context of anticancer therapy. Nat. Rev. Cancer 5, 689-698.

Konopleva, M.Y., and Jordan, C. T. (2011).Leukemia stem cells and microenvironment: biology and therapeutic targeting. J. Clin. Oncol. 29, 591-599.

Kuiken, H. J., and Beijersbergen, R. L. (2010). Exploration of synthetic lethal interactions as cancer drug targets. Future. Oncol. 6, 1789-1802.

Lucchesi, J. C. (1968). Synthetic lethality and semilethality among functionally related mutants of Drosophila melanogaster. Genetics 59, 37-44.

Luo, J., Solimini, N. L., and Elledge, S. J. (2009). Principles of cancer therapy: oncogene and non-oncogene addiction. Cell 136, 823-837.
Rajkumar, S. V., Richardson, P. G., Hideshima, T., and Anderson, K. C. (2005). Proteasome inhibition as a novel therapeutic target in human cancer. J. Clin. Oncol. 23, 630-639.

Saito, Y., Uchida, N., Tanaka, S., Suzuki, N., TomizawaMurasawa, M., Sone, A., Najima, Y., Takagi, S., Aoki, Y. Wake, A., Taniguchi, S., Shultz, L. D., and Ishikawa, F. (2010). Induction of cell cycle entry eliminates human leukemia stem cells in a mouse model of AML. Nat. Biotechnol. 28, 275-280.

Sheikh, K. D., Banerjee, P. P., Jagadeesh, S., Grindrod, S. C., Zhang, L., Paige, M., and Brown, M. L. (2010). Fluorescent epigenetic small molecule induces expression of the tumor suppressor ras-association domain family $1 \mathrm{~A}$ and inhibits human prostate xenograft. $J$. Med. Chem. 53, 2376-2382.

Tennant, D. A., Duran, R. V., and Gottlieb, E. (2010). Targeting metabolic transformation for cancer therapy. Nat. Rev. Cancer 10, 267-277.

Trepel, J., Mollapour, M., Giaccone, G., and Neckers, L. (2010). Targeting the dynamic HSP90 complex in cancer. Nat. Rev. Cancer 10, 537-549.

Warburg, O. (1923). Metabolism of tumors. Biochem. Z. 142, 371-333.

Whitesell, L., and Lindquist, S. L. (2005). HSP90 and the chaperoning of cancer. Nat. Rev. Cancer 5, 761-772.

Zhou, B. B., Zhang, H., Damelin, M., Geles, K. G., Grindley, J. C., and Dirks, P. B. (2009). Tumour-initiating cells: challenges and opportunities for anticancer drug discovery. Nat. Rev. Drug Discov. 8, 806-823.

Received: 14 March 2011; accepted:20 April 2011;published online: 02 May 2011.

Citation: Wang Y and Giaccone G (2011) Challenges in cancer molecular targets and therapeutics. Front. Oncol. 1:4. doi: 10.3389/fonc.2011.00004

This article was submitted to Frontiers in Cancer Molecular Targets and Therapeutics, a specialty of Frontiers in Oncology.

Copyright (c) 2011 Wang and Giaccone. This is an openaccess article subject to a non-exclusive license between the authors and Frontiers Media SA, which permits use, distribution and reproduction in other forums, provided the original authors and source are credited and other Frontiers conditions are complied with. 